\title{
Renal Ewing's sarcoma/primitive neuroectodermal tumor (PNET): a case series of 7 patients and literature review
}

\author{
Lei Liang ${ }^{1 \#}$, Haifeng Song ${ }^{2 \#}$, Binglei Ma ${ }^{1}$, Zhenan Zhang ${ }^{1}$, Kun Zhu ${ }^{1}$, Qinhan Li $^{1}$, Chaohui Zhou ${ }^{1}$, \\ Aolin Li ${ }^{1}$, Jun Liu ${ }^{1}$, Quan Zhang ${ }^{1}$, Shiyu Zhu' ${ }^{1}$, Qian Zhang ${ }^{1}$ \\ ${ }^{1}$ Department of Urology, Peking University First Hospital, Institute of Urology, Peking University, National Urological Cancer Center, Beijing, \\ China; ${ }^{2}$ Department of Urology, Beijing Tsinghua Changgung Hospital, Beijing, China \\ Contributions: (I) Conception and design: L Liang, H Song, Q Zhang; (II) Administrative support: Q Zhang; (III) Provision of study materials or \\ patients: B Ma, Z Zhang, Q Li; (IV) Collection and assembly of data: J Liu, S Zhu, Q Zhang; (V) Data analysis and interpretation: K Zhu, C Zhou, \\ A Li; (VI) Manuscript writing: All authors; (VII) Final approval of manuscript: All authors. \\ \#These authors contributed equally to this work. \\ Correspondence to: Qian Zhang. Department of Urology, Peking University First Hospital, Institute of Urology, Peking University, National \\ Urological Cancer Center, Beijing, China. Email: zhangqianbjmu@126.com.
}

\begin{abstract}
Background: Primitive neuroectodermal tumor (PNET) is a rare kind of sarcoma that is primarily found in the kidney and has a very poor prognosis. Here, we review and summarize the clinical data of patients with renal PNET in our center and follow up the patients for survival status. Although the current literature suggests that chemotherapy may benefit the survival of these patients, the information from our center suggests that this may not be the case.

Methods: We retrospectively analyzed the clinical data of patients with renal PNET diagnosed pathologically at Peking University First Hospital from January 1, 2007, to January 1, 2018. All of the patients were followed up for survival status.

Results: Seven patients with renal PNET were found. The ratio of males to females was 6:1. The median age was 29 years (21-72 years) at the time of diagnosis. The preoperative imaging examination showed a large renal mass protruding outwards from the renal contour, with internal necrosis and hemorrhage. Six/7 patients were diagnosed with distant metastasis or retroperitoneal lymph node metastasis. The main clinical manifestations of patients were pain (5/7) and fever (3/7). In immunohistochemistry, all patients' samples were CD99 positive. All patients died in our follow-up, with an average overall survival (OS) of 12.09 months (1.90-26.77 months).
\end{abstract}

Conclusions: As a rare renal tumor, renal PNET has a propensity to occur in young males. Most patients have distant metastasis when they are diagnosed, and the prognosis is very poor. Effective treatments are urgently needed.

Keywords: Renal primitive neuroectodermal tumor (renal PNET); renal; case series

Submitted Jul 27, 2020. Accepted for publication Nov 24, 2020.

doi: $10.21037 /$ tau-20-1122

View this article at: http://dx.doi.org/10.21037/tau-20-1122

\section{Introduction}

As a member of the Ewing's sarcoma family of tumors (ESFTs), primitive neuroectodermal tumors (PNETs) are a rare kind of sarcoma, especially those found as primary tumors in the kidney, and fewer than 200 cases have been reported thus far. Renal PNET is more common in young adults and older children (median age: 29 years old, 4-61 years old), and the male-to-female incidence ratio is 2:1-3:1. Renal PNET has a very poor prognosis; the 2-year survival rate of patients after radical nephrectomy was $80 \%$ and that of patients without radical nephrectomy was $30 \%$ (1). Forty percent of patients had complications 
with tumor thrombosis and distant metastasis at diagnosis, with a median disease-free survival (DFS) time of 5 months and a median survival time of 24 months $(2,3)$. The clinical manifestations and imaging findings of renal PNET are often nonspecific compared with those of other renal cell carcinomas. Renal PNET also has no specificity in imaging examinations; it often manifests as a single, large mass without obvious boundaries in CT, MRI or ultrasonography (4). The diagnosis of PNET is still based on the histological and immunohistochemical staining of biopsy or surgical specimens; $90 \%$ of patients with PNET had a specific translocation gene, $\mathrm{t}(11: 22)$ (q24; q12), and the fusion gene EWS-Fli-1, which could be found by fluorescence in situ hybridization (FISH) and reverse transcription polymerase chain reaction (RT-PCR), which can help in diagnosis (5). However, there is still no consensus on the best treatment for such a rare disease. Surgery is currently the main treatment strategy, especially radical nephrectomy. Adjuvant radiotherapy is also required for patients with positive margins or late-stage disease, and neoadjuvant and adjuvant chemotherapy may prolong survival in patients with metastases $(1,3,6)$.

Here, we review and summarize the clinical data of patients with renal PNET diagnosed pathologically in our center from January 1, 2007, to January 1, 2018, and follow up the patients for survival status. We present the following article in accordance with the AME Case Series reporting checklist (available at: http://dx.doi. org/10.21037/tau-20-1122).

\section{Methods}

\section{Clinical data collection and follow-up}

We retrospectively analyzed the clinical data of patients with renal PNET diagnosed pathologically through aspiration biopsy or surgery at Peking University First Hospital from January 1, 2007, to January 1, 2018. The patients' specimens were fixed by formalin and embedded in paraffin for HE staining and immunohistochemical analysis. All pathological slices were read by two pathologists, and the results were reviewed by a senior pathologist.

Two researchers collected clinical data, including patient age, sex, symptoms, imaging data (including whether there was tumor thrombosis or distant metastasis) and immunohistochemical results. After two copies of the data were collected, the third researcher reviewed and collated the data.
All patients were followed up by telephone. In June 2020, the follow-up treatment (chemotherapy or not) and overall survival (OS) were recorded. All the follow-up visits were completed by the same researcher, and telephone follow-up of each patient's family members was conducted. All followup visits were conducted for a second time 1 week later. For patients with inconsistent results from the two followup visits, we conducted a third telephone follow-up after a period of time. In addition, our telephone follow-up strictly followed a set sequence and question content.

All procedures performed in this study were in accordance with the Declaration of Helsinki (as revised in 2013). This study was approved by the Research Ethics Committee of Peking University First Hospital, Peking University (approval number 2020-725). All patients' family members were informed, and all agreed to the use of the patients' clinical data in this retrospective study.

\section{Statistical analysis}

OS is expressed as the average and range. Other results including patient age, sex, symptoms, imaging data whether there was tumor thrombosis or distant metastasis) and immunohistochemical results are expressed as numbers and percentages. The statistical analysis was conducted by Microsoft Excel 2013.

\section{Results}

Through a retrospective analysis of the medical records of the Peking University First Hospital from January 1, 2007, to January 1, 2018, we found 7 patients with renal PNET diagnosed pathologically. Their clinical data are summarized in Table 1.

Among them, 6 patients were male, and 1 patient was female. The median age was 29 years (21-72 years). The preoperative imaging examinations of patients were different, but all of them showed a large renal mass, protruding outwards from the renal contour, some of which were accompanied by internal necrosis and hemorrhage (Figure 1). The CT scan of case 3 showed a renal mass protruding outwards from the renal contour, with internal necrosis and hemorrhage. Four patients were diagnosed with distant metastasis (including lung, bone or liver metastases), and two patients had retroperitoneal lymph node metastasis.

In terms of symptoms, the main clinical manifestation of most patients was pain (5/7). The second most common 
Table 1 Clinical features of 7 patients with renal primitive neuroectodermal tumor

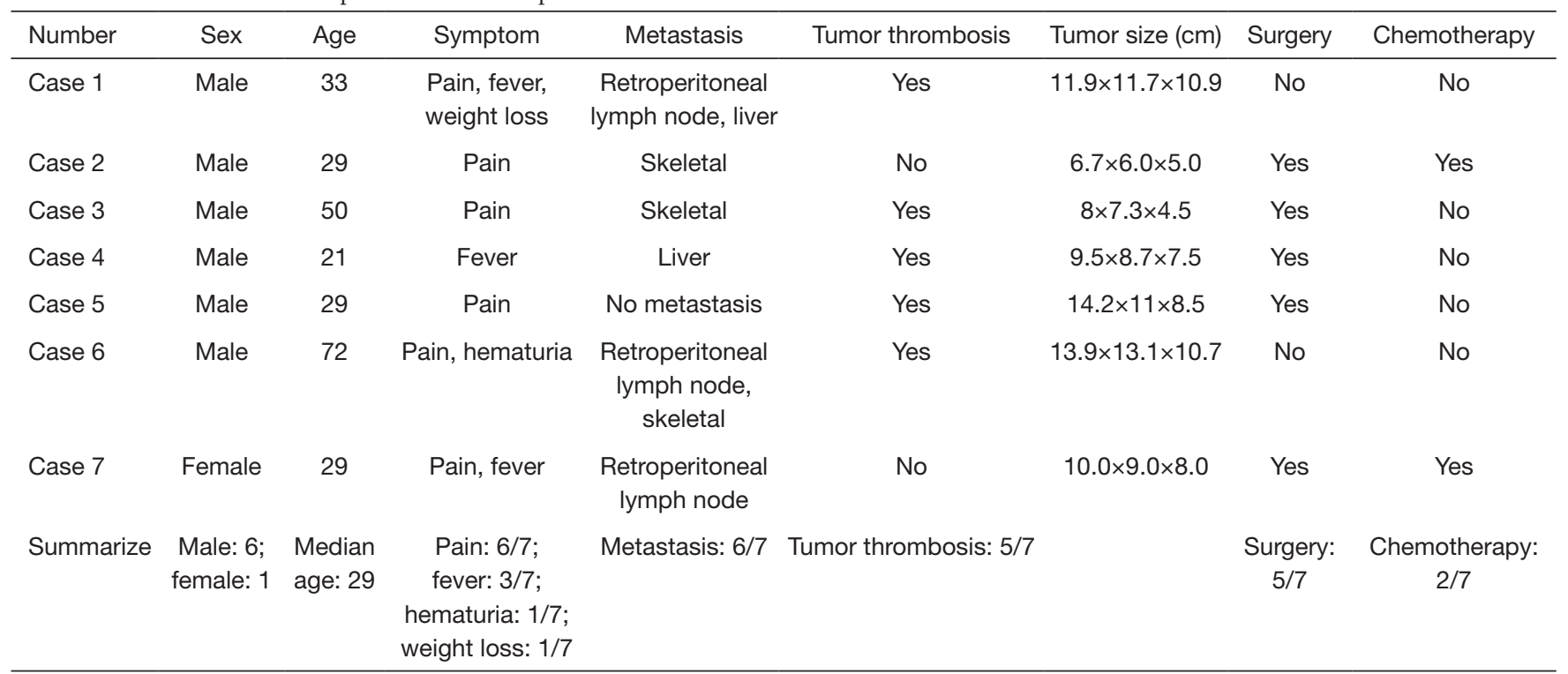

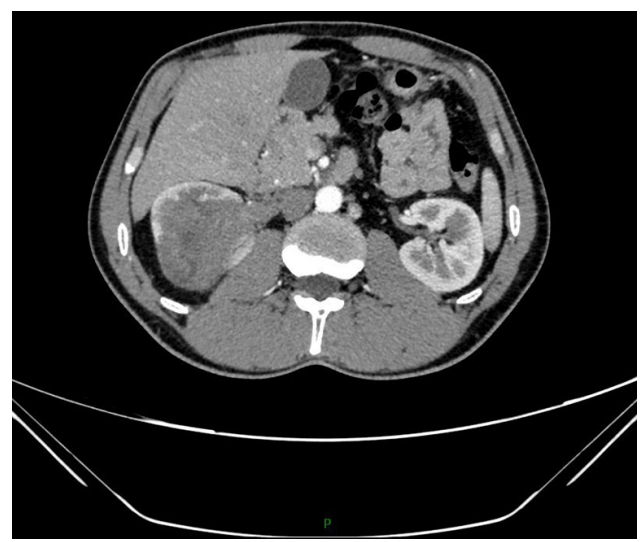

Figure 1 CT of case 3, shows a renal mass, protruding outwards the renal contour, with internal necrosis and hemorrhage.

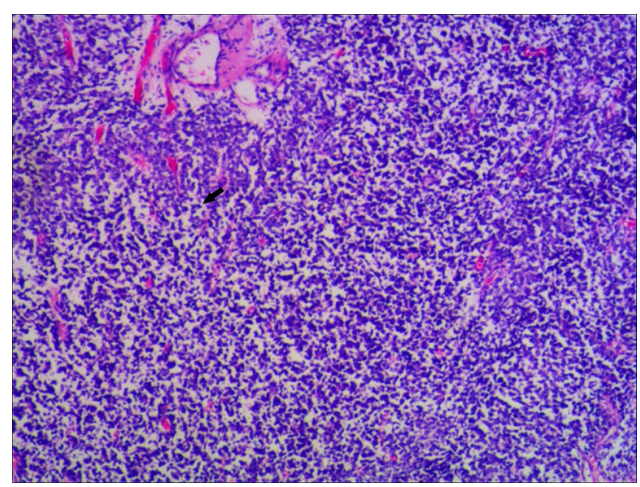

Figure $2 \mathrm{HE}$ stain of case 5's sample, shows a dens mass of small round tumor cells (arrow). Magnification: 200×. symptom was fever (3/7). Among the 3 patients with fever, case 1 showed a persistent high fever $\left(39^{\circ} \mathrm{C}\right)$, and cases 4 and 7 showed persistent low fever (below $38^{\circ} \mathrm{C}$ ).

Case 1 did not receive surgical treatment due to persistent high fever and a deterioration of the general condition after needle biopsy of the kidney; this patient died very quickly. Case 6 refused further surgical treatment after the biopsy diagnosis of PNET.

Two patients (case 2 and case 7) received postoperative chemotherapy, but case 2 completed only three rounds of chemotherapy due to bone marrow suppression after chemotherapy, and case 7 did not complete the chemotherapy because of an inability to tolerate the side effects.

In the $\mathrm{HE}$ staining, a dense mass of small round tumor cells was observed (Figure 2). HE staining of the sample from case 5 showed a dense mass of small round tumor cells, and typical Homer-Wright rosettes were observed in 2 specimens: 7/7 were CD99 positive and LCA negative, 6 patients were VIM positive, and $4 / 7$ patients were NSE positive. Five patients were tested for CgA, but only 1 case was positive, and 3 cases were tested for WT1, and all of them were negative. The results of other immunohistochemical markers are shown in Table 2 and Figure 3.

All patients were followed up by telephone, and all patients had died, with a median survival time of 11.47 months. Their survival status is summarized in Table 3. The only patient without distant metastasis at diagnosis had 
Table 2 Immunohistochemistry markers of 7 patients with renal primitive neuroectodermal tumor

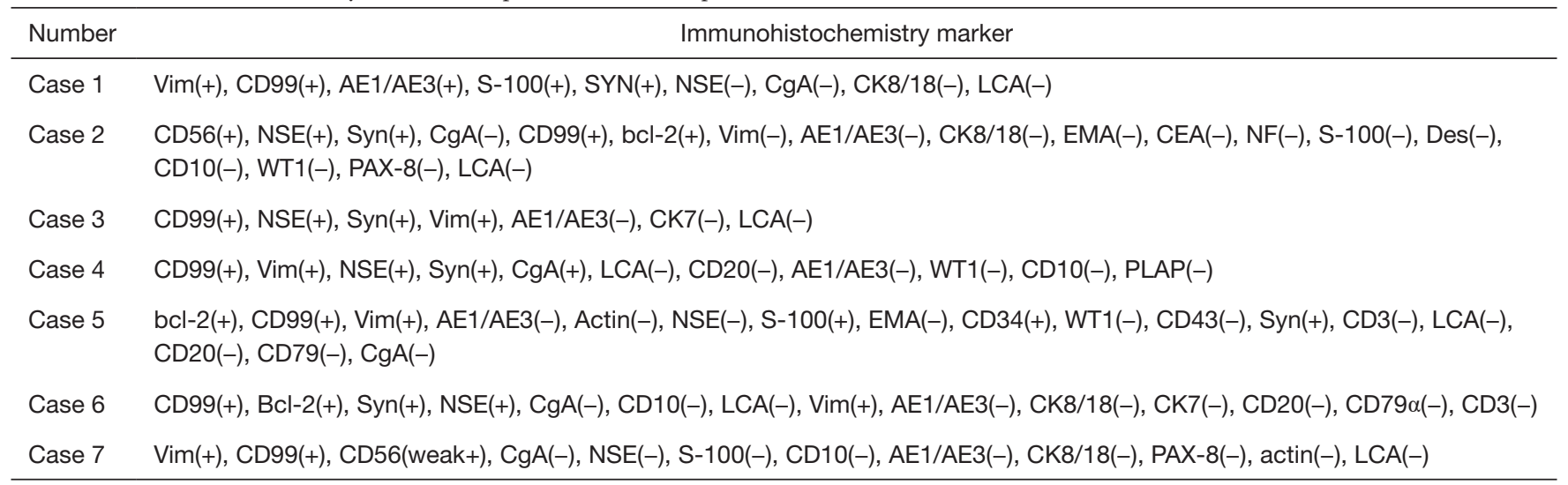

CK, cytokeratin; DES, desmin; EMA, epithelial membrane antigen; NF, neurofilament; NSE, neuron-specific enolase; Syn, synaptophysin; VIM, vimentin; WT-1, Wilms tumor protein; CD, cluster of differentiation; CgA, chromogranin A; CEA, carcinoembryonic antigen; LCA, leukocyte common antigen ; BCL2,B-cell lymphoma 2 ; PLAP, placental alkaline phosphatase;

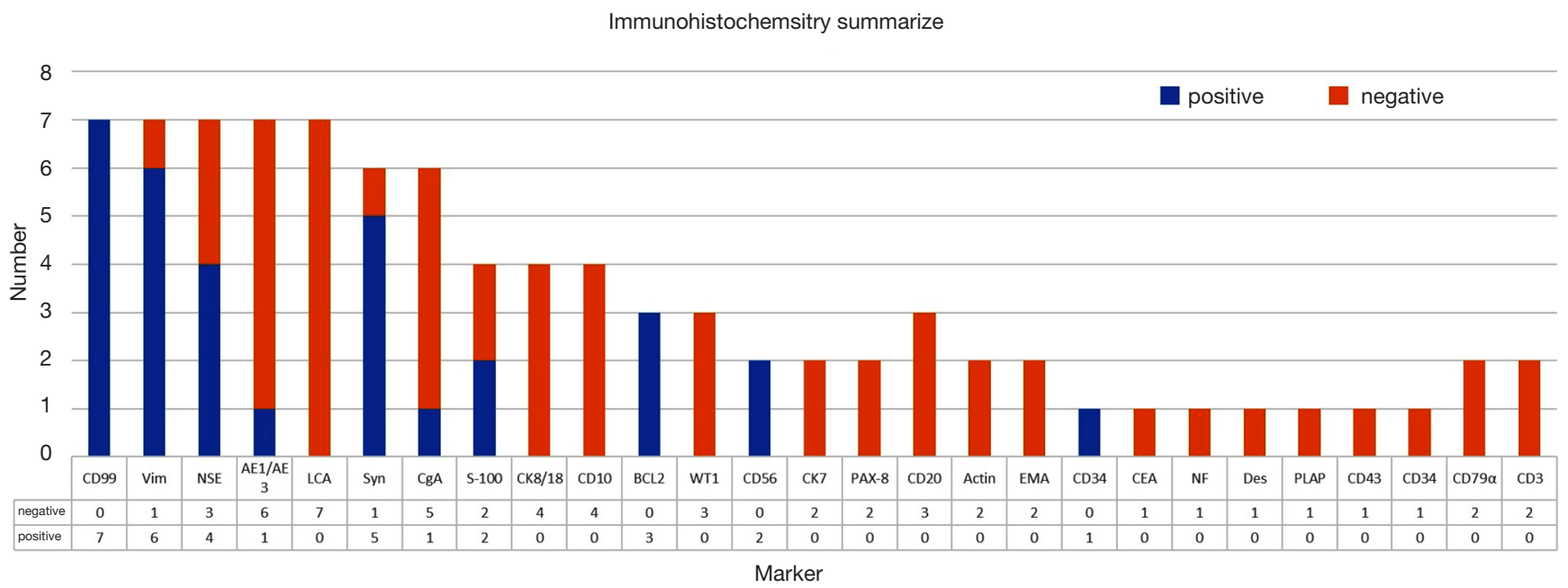

Figure 3 Summary of the immunohistochemistry markers.

Table 3 Survival analysis of 7 patients with renal primitive neuroectodermal tumor

\begin{tabular}{lccc}
\hline \multirow{2}{*}{ Variable } & Case & \multicolumn{2}{c}{ OS (months) } \\
\cline { 3 - 4 } & & Average & Range \\
\hline Total & 7 & 12.09 & $1.90-26.77$ \\
Metastasis & & & \\
Yes & 6 & 9.64 & $1.90-16.17$ \\
No & 1 & 26.77 & - \\
Chemotherapy & & & $5.40-11.47$ \\
Yes & 2 & 8.43 & $1.90-26.77$ \\
No & 5 & 13.55 & \\
\hline
\end{tabular}

the longest OS (26.77 months). The average OS of the two patients receiving chemotherapy was 8.43 months, which was lower than that of the patients who did not receive chemotherapy (13.55 months). Due to the small number of patients in our cohort, we could not make a statistical comparison.

\section{Discussion}

We retrospectively summarized the medical records of 7 patients with renal PNET diagnosed in our center. The median age was 29 years (21-72 years). Four out of 7 patients were diagnosed with distant metastasis 
(including lung, bone or liver metastases), and 2 out of 7 patients had retroperitoneal lymph node metastasis. The main clinical manifestations were pain and fever. The median survival time was 11.47 months. The average OS of the two patients receiving chemotherapy was 8.43 months, which was lower than that of the patients who did not receive chemotherapy (13.55 months).

PNET is a kind of malignant small round cell tumor originating from the neural crest that belongs to the Ewing's sarcoma family of tumors (ESFTs). PNET is common in the central nervous system, rib and paravertebral region, including the skin, soft tissue and lung. Renal PNET is extremely rare, and fewer than 200 cases have been reported worldwide. In the reported cases, renal PNET was more common in young adults and older children (median: 29 years old, 4-61 years old), and the male-to-female incidence ratio was 2:1-3:1 (7-9).

The clinical features of renal PNET are not specific compared with those of other types of renal tumors. The typical "renal cancer triad syndrome" (low back pain, hematuria and abdominal mass) can be observed in renal PNET. In addition, irregular fever and weight loss can be clinical manifestations (1). Renal PNET also has no specificity in imaging examinations; it often manifests as a single, large mass without obvious boundaries in CT, MRI or ultrasonography. However, compared with renal cell carcinoma, the boundary of renal PNET on contrastenhanced CT is more fuzzy; internal necrosis and separation are more common and more likely to invade the renal vein and inferior vena cava; and the enhancement degree of PNET in the cortical and parenchymal phases is relatively small $(4,10)$. In MRI, PNET was described as isointense or low signal on T1WI and inhomogeneous medium to high signal on T2WI $(10,11)$.

The diagnosis of renal PNET should be made by histological and immunohistochemical staining. The product of the $M I C-2$ gene, CD99, is usually strongly positive, and neuron-specific enolase (NSE) and synaptophysin (Syn) may also be positive in PNET (12). The differential diagnosis of renal PNET includes neuroblastoma, adult Wilms' tumor, malignant lymphoma, rhabdomyosarcoma, small-cell carcinoma and synovial sarcoma (monophasic, poorly differentiated, round cell variant). Immunohistochemical markers for differential diagnosis are cytokeratin (CK; for Wilms' tumor, smallcell carcinoma and synovial sarcoma), leukocyte common antigen (LCA; for lymphoma), NSE/chromogranin A (NSE/CgA; for neuroblastoma) and CD-99 (for PNET).
Although tumor cells can show NSE positivity in some PNET cases, as seen in neuroblastoma, CD99 is usually negative in neuroblastoma (13). In addition, the typical histological features of PNET, Homer-Wright rosettes, are rare in other extraosseous Ewing's sarcomas, which is helpful for the diagnosis of PNET. Further, $90 \%$ of PNET patients had a specific translocation gene, $\mathrm{t}$ (11:22) (q24; q12), and the fusion gene EWS-Fli-1, which could be found by fluorescence in situ hybridization (FISH) and reverse transcription polymerase chain reaction (RT-PCR) (5,13-19). In our 7 patients, all patients' samples were positive for CD99, which is a typical immunohistochemical feature of ES/PNET. In addition, all patients' samples were negative for LCA, which well differentiates PNET from lymphoma. To identify other epithelial tumors, all our specimens had negative CK8/18, CK7 or AE1/AE3 results. Some patients' specimens were NSE positive (4/7) and CgA positive (1/5). Unfortunately, our center was unable to perform FISH or RT-PCR to confirm the presence of the EWS-FLI1 fusion gene in these patients.

The 5-year survival rate of PNET is only $45-55 \%$, and the median recurrence-free survival (RFS) is only 2 years for advanced PNET $(1,20)$. Similar to other PNETs, renal PNETs are high-grade malignant. Approximately $40 \%$ of patients have distant metastasis when diagnosed. The common metastasis locations include the regional lymph nodes, liver and lung. Risi et al. summarized the clinical data of 116 reported renal PNET patients in 2012. For patients with distant metastasis, the median DFS was only 5 months, and the 18 -month survival rate was only $60 \%$, which was significantly lower than that of patients without metastasis (85\%) (1).

Patients usually need a combination of multiple treatment strategies, including radical nephrectomy and chemotherapy (VAC (cyclophosphamide + adriamycin + vincristine) and IE (ifosfamide + etoposide) are most common), and adjuvant radiotherapy is also required for patients with positive margins or advanced stage, but in existing studies, there was no statistically significant survival benefit for patients receiving adjuvant therapy before or after surgery compared to patients receiving surgery alone (12-month OS was $93 \%$ compared to $75 \%, \mathrm{P}=0.092)(1,21)$. In addition, with further research on the Ewing sarcoma family and the EWS-FLI1 fusion gene, some drugs aimed at the signal transduction pathways involved in this gene, such as integrin, Wnt, IGF, EGF and PDGF, are also emerging gradually. However, whether these drugs can eventually enter clinical practice and improve the prognosis of patients 
is still unknown $(9,22)$. In our results, the survival of patients was worse than that reported in the literature. The average OS time was only approximately 1 year. This may be related to the fact that most of our patients had distant metastasis $(6 / 7)$ at diagnosis, and most patients did not receive chemotherapy (5/7). In addition, the only 2 patients who received chemotherapy did not complete the chemotherapy due to the side effects caused by the drugs. Furthermore, unfortunately, because of the time that had passed between chemotherapy and our telephone follow-up, the patients' families were unable to provide details on the chemotherapy regimen, so we were unable to conduct further analysis about this question. And due to the existence of literature bias, clinicians are often more willing to report successful treatment of their patients, with more successful operations and longer survival times. Therefore, some patients who are also diagnosed with this disease but have poor prognosis may not be reported. These factors create a possible bias in the literature. All of these factors led to the OS time of our patients being lower than that reported in the previous literature.

There are several limitations in our study: (I) this is a retrospective study, and the number of cases is low. This creates a potential bias, although for such a rare type of renal tumor, a prospective study is very difficult, and prospective clinical trials are undoubtedly of greater significance for diagnosis and treatment. (II) Because our patients come from all over the country and the disease is very rare, there is usually only one patient with the disease in a certain region. It is very difficult for us to follow up with patients and their families with paperbased follow-up forms. Therefore, we chose telephone follow-up, which creates a potential risk of bias. (III) Since postoperative treatment was not conducted in our hospital, and it had been some time since the death of the patients when the patients' family members were contacted, the family members of the only two patients who received postoperative chemotherapy could not recall the chemotherapy plan and cycle administered to the patients.

Since the first report of renal PNET in 1975, people's understanding of this disease has gradually deepened (23). According to the current reports, renal PNET is still a malignant tumor with extremely poor prognosis, and although chemotherapy is currently considered a potentially effective treatment, the side effects of chemotherapy are still extremely painful for patients, and better standardized treatment regimens are still needed to prolong survival.

\section{Conclusions}

Renal PNET, as a rare high-grade malignant tumor, has a poor prognosis. Most patients have distant metastasis and venous tumor thrombosis. Although the existing research shows that surgery combined with chemotherapy may be beneficial to the OS of patients, the poor general condition of patients with distant metastasis often makes them unable to tolerate the side effects of chemotherapy. Whether these advanced patients with poor general condition can benefit from chemotherapy needs more research.

\section{Acknowledgments}

Funding: None.

\section{Footnote}

Reporting Checklist: The authors have completed the AME Case Series reporting checklist. Available at http://dx.doi. org/10.21037/tau-20-1122

Data Sharing Statement: Available at http://dx.doi. org/10.21037/tau-20-1122

Peer Review File: Available at http://dx.doi.org/10.21037/ tau-20-1122

Conflicts of Interest: All authors have completed the ICMJE uniform disclosure form (available at http://dx.doi. org/10.21037/tau-20-1122). QZ serves as an unpaid editorial board member of this journal. The other authors have no other conflicts of interest to declare.

Ethical Statement: The authors are accountable for all aspects of the work in ensuring that questions related to the accuracy or integrity of any part of the work are appropriately investigated and resolved. All procedures performed in this study were in accordance with the Declaration of Helsinki (as revised in 2013). This study was approved by the Research Ethics Committee of Peking University First Hospital, Peking University (approval number 2020-725). All patients' family members were informed, and all agreed to the use of the patients' clinical data in this retrospective study.

Open Access Statement: This is an Open Access article distributed in accordance with the Creative Commons 
Attribution-NonCommercial-NoDerivs 4.0 International License (CC BY-NC-ND 4.0), which permits the noncommercial replication and distribution of the article with the strict proviso that no changes or edits are made and the original work is properly cited (including links to both the formal publication through the relevant DOI and the license). See: https://creativecommons.org/licenses/by-nc-nd/4.0/.

\section{References}

1. Risi E, Iacovelli R, Palazzo A, et al. Chemotherapy is effective in Primitive Neuroectodermal Tumor (PNET)/ Ewing Sarcoma (EWS) of the kidney. Ann Oncol 2012;23:488.

2. Giliberto GL, Di Franco CA, Rovereto B. Primitive neuroectodermal tumour of kidney with thrombosis of the inferior vena cava and good responsive to surgical and medical treatment: description of a case and revision of literature. Urologia 2018;85:127-9.

3. Goel V, Talwar V, Dodagoudar C, et al. Primary renal primitive neuroectodermal tumor. J Postgrad Med 2015;61:126-8.

4. Kumar P, Singh A, Deshmukh A, et al. Qualitative and quantitative CECT features for differentiating renal primitive neuroectodermal tumor from the renal cell carcinoma and its subtypes. Br J Radiol 2019;92:20180738.

5. Kang SH, Perle MA, Nonaka D, et al. Primary Ewing sarcoma/PNET of the kidney: Fine-needle aspiration, histology, and dual color break apart FISH assay. Diagn Cytopathol 2007;35:353-7.

6. Kermani AT, Ghorbani H, Gharib M, et al. Unilateral Renal Primitive Neuroectodermal Tumor (PNET). International Journal of Cancer Management 2017;10.

7. Mandal PK, Mukherjee S, Roy S, et al. PNET of kidney: Report of four cases. Indian J Med Paediatr Oncol 2012;33:130-3.

8. Agarwal K, Bhushan R, Yadav PS. Primary Large Cell (Atypical) Ewing Sarcoma/Primitive Neuroectodermal Tumour of Kidney. J Clin Diagnostic Res 2018;12:ED5-7.

9. Bartholow T, Parwani A. Renal primitive neuroectodermal tumors. Arch Pathol Lab Med 2012;136:686-90.

10. Akkaya Z, Peker E, Gulpinar B, et al. CT and MRI Findings in a Rare Case of Renal Primitive Neuroectodermal Tumor. Pol J Radiol 2016;81:401-6.

11. Zheng Z, Li P, Ma T, et al. Primary Pediatric Renal Primitive Neuroectodermal Tumor: A Case Report With Different Stage CT and MRI Images. Urology 2020;136:241-4.
12. Gali SC, Kumarguru BN, Bhat B, et al. Renal Primitive Neuroectodermal Tumour: Case Report of a Rare Entity. J Clin Diagn Res 2017;11:ED17-9.

13. Popov SD, Sebire NJ, Popova ED, et al. Primitive neuroectodermal tumor of the kidney in children; its differential diagnosis with Wilms tumor. Arkh Patol 2009;71:41-3.

14. Miao C, Yang J, Xue J, et al. Renal Ewing sarcoma/ primitive neuroectodermal tumor in a pregnant woman who underwent robot-assisted laparoscopic nephrectomy: a case report and literature review. Onco Targets Ther 2018;11:6839-43.

15. Etani T, Naiki T, Ando R, et al. A Case of Renal Primitive Neuroectodermal Tumor Confirmed by Fluorescence in situ Hybridization. Case Rep Oncol 2015;8:205-11.

16. Kuroda $M$, Urano $M$, Abe $M$, et al. Primary primitive neuroectodermal tumor of the kidney. Pathol Int 2000;50:967-72.

17. Sheaff M, McManus A, Scheimberg I, et al. Primitive Neuroectodermal Tumor of the Kidney Confirmed by Fluorescence In Situ Hybridization. Am J Surg Pathol 1997;21:461-8.

18. Takeuchi T, Iwasaki H, Ohjimi Y, et al. Renal primitive neuroectodermal tumor: a morphologic, cytogenetic, and molecular analysis with the establishment of two cultured cell lines. Diagn Mol Pathol 1997;6:309-17.

19. Sheaff M, Scheimberg I, McManus A, et al. Primitive neuroectodermal tumour of the kidney confirmed by detection of chromosome 22q12 translocation using fluorescence in situ hybridisation. J Pathol 1996;178:46A.

20. Pomara G, Cappello F, Cuttano MG, et al. Primitive Neuroectodermal Tumor (PNET) of the kidney: a case report. BMC Cancer 2004;4:3.

21. Citak EC, Oguz A, Karadeniz C, et al. Primitive neuroectodermal tumor of the kidney in a child. Pediatr Hematol Oncol 2009;26:481-6.

22. Balamuth NJ, Womer RB. Ewing's sarcoma. Lancet Oncol 2010;11:184-92.

23. Seemayer TA, Thelmo WL, Bolande RP, et al. Peripheral Neuroectodermal Tumors. Perspect Pediatr Pathol 1975;2:151-72.

Cite this article as: Liang $\mathrm{L}$, Song $\mathrm{H}, \mathrm{Ma} \mathrm{B}$, Zhang Z, Zhu K, Li Q, Zhou C, Li A, Liu J, Zhang Q, Zhu S, Zhang Q. Renal Ewing's sarcoma/primitive neuroectodermal tumor (PNET): a case series of 7 patients and literature review. Transl Androl Urol 2021;10(2):548-554. doi: 10.21037/tau-20-1122 